\section{Science and Mexico are the losers in institute politics}

\section{Events at Mexico's Instituto}

Potosino de Investigación

Científica y Tecnológica (IPICYT)

have escalated to crisis point (see page 148). We the undersigned call on the world's academic community to help reverse the damage currently being done in this research institution, once a shining example for all developing nations.

After battling for two years, Humberto and Mauricio Terrones - acclaimed leaders of IPICYT's prestigious nanoscience and nanotechnology group - have been removed from office. This flies in the face of the presumed commitment of the Consejo Nacional de Ciencia y Tecnología (CONACYT), Mexico's highest research authority, to seek a "solution that will be a product of open negotiations carried out with tolerance, good will, great objectivity and agreements that avoid personal aggression and radicalization of positions". This is the wording of a recommendation solicited by CONACYT from a prestigious group of scientists formed to help prevent this outcome.

An international group of some 75 scientists has been working hard with us for two years to broker a solution to this sorry affair, to no avail. Our hope is that President Felipe Calderón will step in and avert further damage. Otherwise, the prestige of Mexico's science and the prospects for its technological development will suffer, as young Mexican scientists won't return after being trained in research abroad.

The academic community should join forces to reverse this situation.

Harold W. Kroto Florida State University, USA

e-mail: kroto@chem.fsu.edu

Pulikel M. Ajayan Rice University,

Texas, USA

Anthony K. Cheetham University of Cambridge, UK

\section{Mildred S. Dresselhaus Massachusetts Institute of Technology, USA Morinobu Endo Shinshu University, Japan \\ Alan L. Mackay Birkbeck College, University of London, UK Ljubisa R. Radovic Pennsylvania State University, USA \\ Colour-coded targets would help clarify biodiversity priorities}

In this International Year of Biodiversity, we should be setting ambitious but realistic targets for biodiversity policy over the next ten years. Those shaped at last month's sixth Trondheim Conference on Biodiversity in Norway will be refined by the Subsidiary Body on Scientific, Technical and Technological Advice in May and at the Conference of the Parties of the Convention on Biological Diversity (CBD) in October.

As participating scientists in the international biodiversity programme DIVERSITAS, we welcome the draft set of 2020 targets proposed by the CBD. But the targets continue to mix the biodiversity we value highly (that is, the conservation agenda) and the biodiversity we urgently need to secure the benefits people derive from fully functioning ecosystems.

To resolve competing demands, these different priorities should be made explicit by categorizing the targets according to their primary motivation. We suggest the use of red targets to stem urgent deleterious biodiversity loss, green targets for conservation priorities and blue targets to secure the long-term benefits from functioning ecosystems.

The CBD should work closely with the science community to develop these targets for changing environments and in the light of new scientific discoveries. The proposed Intergovernmental Platform for Biodiversity and Ecosystem Services (H. Mooney and G. Mace Science 325,
1474; 2009) and the global biodiversity observation system GEO BON (R. J. Scholes et al. Science 321, 1044-1045; 2008) will be valuable tools in this collaboration.

Anne Larigauderie DIVERSITAS, 57 Rue Cuvier-CP 41, 75231 Paris Cedex 05, France

e-mail:anne@diversitas-international.org

Georgina M. Mace Imperial College London, Centre for Population Biology, Silwood Park, Ascot SL5 7PY, UK Harold A. Mooney Department of Biology, Stanford University, Stanford, California 94306, USA

\section{Barriers to carbon capture and storage may not be obvious}

There's more to 'Buried trouble' than whether carbon dioxide should be injected under urban areas or offshore (Nature 463, 871-873; 2010). Some barriers to carbon-sequestration measures are less immediately noticeable than public opinion.

For example, the technology should be incorporated into developing energy systems. In most scenarios produced by the Intergovernmental Panel on Climate Change (IPCC), much larger volumes of $\mathrm{CO}_{2}$ will be captured in China and India by 2050 than in developed countries. But the scale and pace of energysystems development, and the necessary carbon capture and storage (CCS) technology transfers, are daunting.

People optimistic about CCS technology transfer to developing countries should remember that transferring even cost-saving technologies (transgenic seeds, for example) has been difficult. We should develop transfer incentives by recognizing CCS investments within the Clean Development Mechanism of the Kyoto Protocol. We must also limit uncertainties surrounding CCS investments in developing countries, particularly in protecting intellectual property in capture technologies.
Another barrier to CCS is

that countries with a weak manufacturing capability are not in a strong position to develop lucrative carbon capture technologies. The Australian government, for example, has committed Aus $\$ 2.4$ billion (US $\$ 2.2$ billion) to its CCS Flagships Programme. But two of the projects rely on capture technologies from Japan (Mitsubishi in the ZeroGen Project) and the United States (GE in the Wandoan Power Project). Governments need to balance their desire to support emerging domestic CCS technologies against importing potentially better technologies from abroad.

\section{CCS solutions are also} subject to the vested interests of national politics. In the coalheavy economies of Canada, the United States and Australia, for example, governments promote CCS in their emissionsreduction promises, but they have been reluctant to mandate the technology.

The real barrier to CCS is that, even in enthusiastic countries, the focus is on selling CCS solutions rather than on mandatory CCS deployment. Advocates should commit to a firm timeline for mandatory CCS on all new and retrofitted large emitters.

Frances Bowen International Resource Industries and Sustainability Centre, Haskayne School of Business, University of Calgary, 2500 University Drive NW, Calgary, Alberta T2N 1N4, Canada, and Smith School of Enterprise and the Environment, University of Oxford, UK

e-mail: fbowen@ucalgary.ca

Contributions to Correspondence may be submitted to

correspondence@nature.com.

Please see the Guide to Authors at go.nature.com/cMCHno. Science publishing issues that may be of interest to authors are regularly featured at our blog Nautilus (http://blogs.nature.com/ nautilus), where we welcome comments and debate. 